\title{
A Review on Morphological, Structural and Electrical Characterization Techniques of Thin films
}

\author{
Dr.G.Velmathi \\ School of Electronics Engineering, VIT University, Chennai CampusChennai 600127 \\ velmathi.g@vit.ac.in
}

\begin{abstract}
This review paper explains about the basics and the working of all the equipments involved in the morphological, structural and electrical analysis of deposited thin films the author has used at Indian Institute of Science. Working of XRD, XPS, SEM/TEM and AFM and other electrical characterization techniques have been explained with necessary schematic diagram and the photographs of the equipments.
\end{abstract}

Keyword-XRD, XPS,

\section{INTRODUCTION}

The thin films synthesized by various techniques such as sputtering, evaporation, chemical vapor deposition etc. are typically characterized for structural, chemical composition, and optical/electrical properties. Complete characterization of the films is necessary to evaluate the quality of the films and to provide an insight into the chemistry occurring during the deposition. The morphological, structural and electrical characterization is carried out with a variety of techniques, including

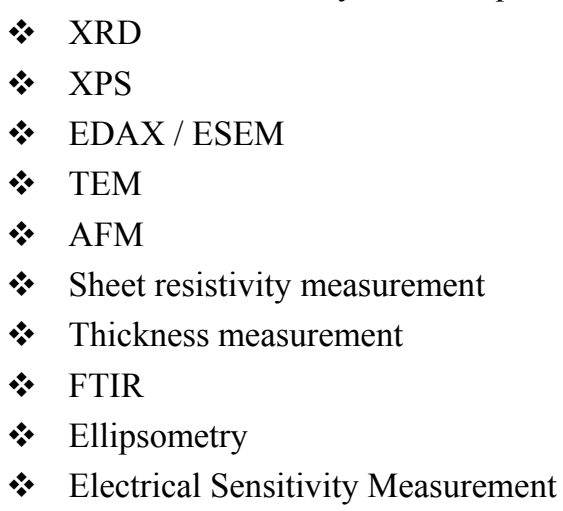

\section{X-RAY DIFFRACTION (XRD)}

$\mathrm{X}$-ray diffraction is a rapid analytical technique used for phase identification of a crystalline material, measurement of sample purity and it can provide information of unit cell dimensions. X-ray diffraction is based on constructive inference of monochromatic X-rays and a crystalline sample. These X-rays are generated by a cathode ray tube, filtered to produce monochromatic radiation, collimated to concentrate, and directed towards the sample. The interaction of the incident rays with the sample produces constructive interferences (and a diffracted ray) when conditions satisfy Bragg's Law [1]

$(\mathbf{n} \boldsymbol{\lambda}=\mathbf{2 d} \sin \boldsymbol{\theta})$ as shown in fig. 1

The Bragg's law relates the wavelength of electromagnetic radiation to the diffraction angle and the lattice spacing in a crystalline sample. These diffracted X-rays are then detected, processed and counted. By scanning the sample through a range of $2 \theta$ angles, all possible diffraction directions of the lattice should be attained due to the random orientation of the powdered material. Conversion of the diffraction peaks to " $\mathrm{d}$ " spacing allows identification of the mineral because each mineral has a set of unique d-spacing. 


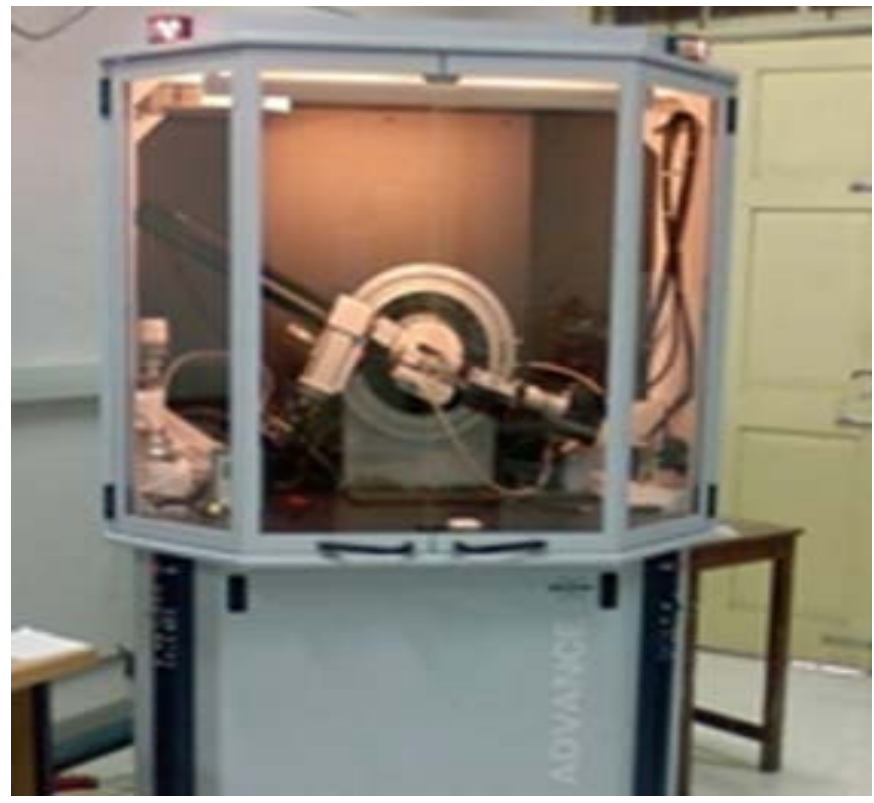

Fig. 1 Photograph of X-ray Diffraction Equipment

This is achieved by comparison of d-spacing with standard reference patterns. A typical X-ray diffractometer as shown in fig.1 consists of three basic components: An X-ray tube, a sample holder, and an X-ray detector.

$\mathrm{X}$-rays are generated in a cathode ray tube by heating a filament to produce electrons, accelerating the electrons towards a target by applying a voltage, and bombarding the target material with electrons. When electrons have sufficient energy to dislodge inner shell electrons of the target material, characteristic X-ray spectra are produced. These spectra consist of several components, the most common being $\mathrm{K}_{\alpha}$ and $\mathrm{K}_{\beta} \cdot \mathrm{K}_{\alpha}$ consists $\mathrm{K}_{\alpha 1}$ and $\mathrm{K}_{\alpha 2} . \mathrm{K}_{\alpha 1}$ has a slightly shorter wavelength and twice the intensity as $\mathrm{K}_{\alpha 2}$. The specific wavelengths are characteristics of the target material $(\mathrm{Cu}, \mathrm{Fe}, \mathrm{Mo}, \mathrm{Cr})$. Filtering, by foils or crystal monochromators, is required to produce monochromatic X-rays needed for diffraction. $\mathrm{K}_{\alpha 1}$ and $\mathrm{K}_{\alpha 2}$ are sufficiently close in wavelength such that a weighted average of the two is used [2-4]. Copper is the most common target material for single-crystal diffraction, with $\mathrm{Cu} \mathrm{K} \mathrm{K}_{\alpha}$ radiation $=1.541 \mathrm{~A}^{\mathrm{O}}$. These X-rays are collimated and directed onto the sample. The collimation is performed using cones or collimators (multi-leaf collimator or iris diaphragms) that are attached directly in front of X-ray tube. As the sample and detector are rotated, the intensity of the reflected X-rays is recorded. When the geometry of the incident X-rays impinging the sample satisfies the Bragg equation, constructive interference occurs and a peak in intensity occurs. A detector records and processes this X-ray signal and converts the signal to a count rate which is then output to a device such as a printer or computer monitor. The geometry of an X-ray diffractometer is such that the sample rotates in the path of the collimated Xray beam at an angle $\theta$ while the X-ray detector is mounted on an arm to collect the diffracted X-rays and rotates at an angle of $2 \theta$. The instrument used to maintain the angle and rotate the sample is termed as goniometer.

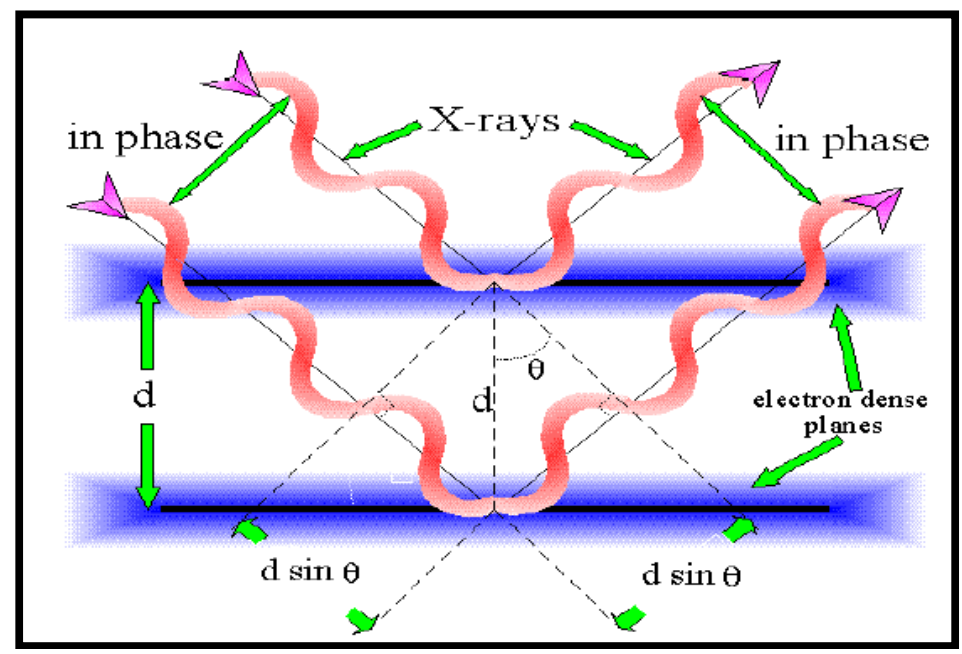

Fig.2 Basic principle involved in diffraction of X-ray beam from assembly of lattice atoms 


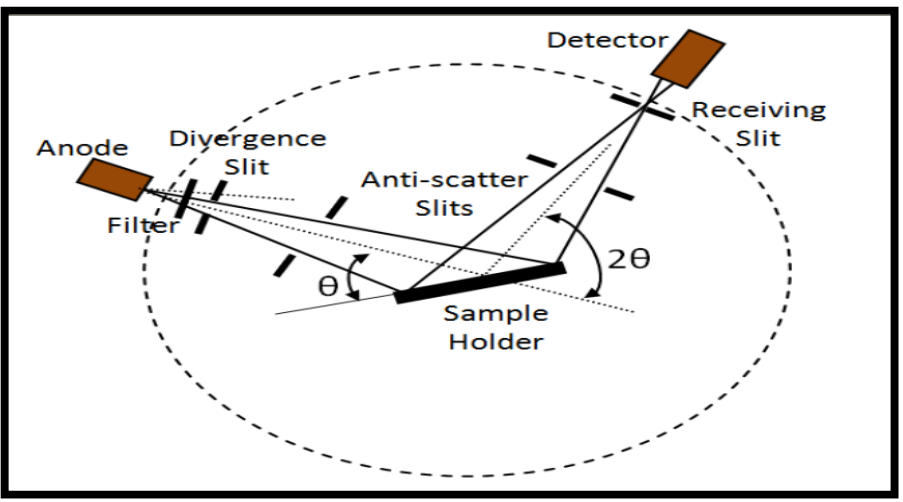

Fig. 3 Schematic of the X-rays experimental setup

Much valuable knowledge about the crystal structure of a material can be obtained from the obtained peak positions, intensity, peak-width and shape. Phase identification is done by comparing the peak position $2 \theta$ values and inter-planar spacing with the known standards JCPDS (joint committee on powder diffraction standards) files supplied by XRD measuring systems suppliers. To determine the strain in the structure the shift in the position of the diffracted peaks is taken into account. In addition, having measured the full widths at half maximum (FWHM) of diffracted peaks, it is possible to calculate the grain size of crystals through the Scherer's formula [1]

$$
d=\frac{0.9 \lambda}{\beta \cos \theta}
$$

where $\beta, \theta$, and $\lambda$ are FWHM, Bragg's angle, and wavelength of the X-rays, respectively. Moreover, XRD is also used for the determination of the thickness of thin films by measuring the space between the primary and secondary or subsidiary maxima diffraction peaks [2].

\section{X-RAY PHOTOELECTRON SPECTROSCOPY (XPS)}

$\mathrm{X}$-ray photoelectron spectroscopy, also known as electron spectroscopy for chemical analysis (ESCA), is a powerful surface-sensitive technique for the determination of chemical composition of materials. It is accomplished by irradiating a sample with X-rays for the excitation of the material and energy analyzing of emitted photoelectrons. Fig.4 shows the XPS equipment used at SSCU, IISc.

The source used for the soft X-rays are generally $\operatorname{MgK} \alpha(1253.6 \mathrm{eV})$ or AlK $\alpha(1486.6 \mathrm{eV})$. Depending on the matrix of atoms in structure of materials, incident X-rays can travel in solids from 1 to $10 \mathrm{~nm}$. The kinetic energy $\left(E_{k}\right)$ of the emitted photo-electron can be calculated through the Einstein photoelectronic relation; $E_{b}=$ $h_{v}-E_{k}-W$. Here, $E_{b}$ is the binding energy (a measure of energy strength of interaction between electron and atom's nuclear charge) of the electron to a particular atom in the material, $\mathrm{h}_{v}$ is the incoming photon energy, and $\mathrm{W}$ is the spectrometer work function [4-6]. It is possible to evaluate the binding energy of an electron on condition that its kinetic energy is known. 


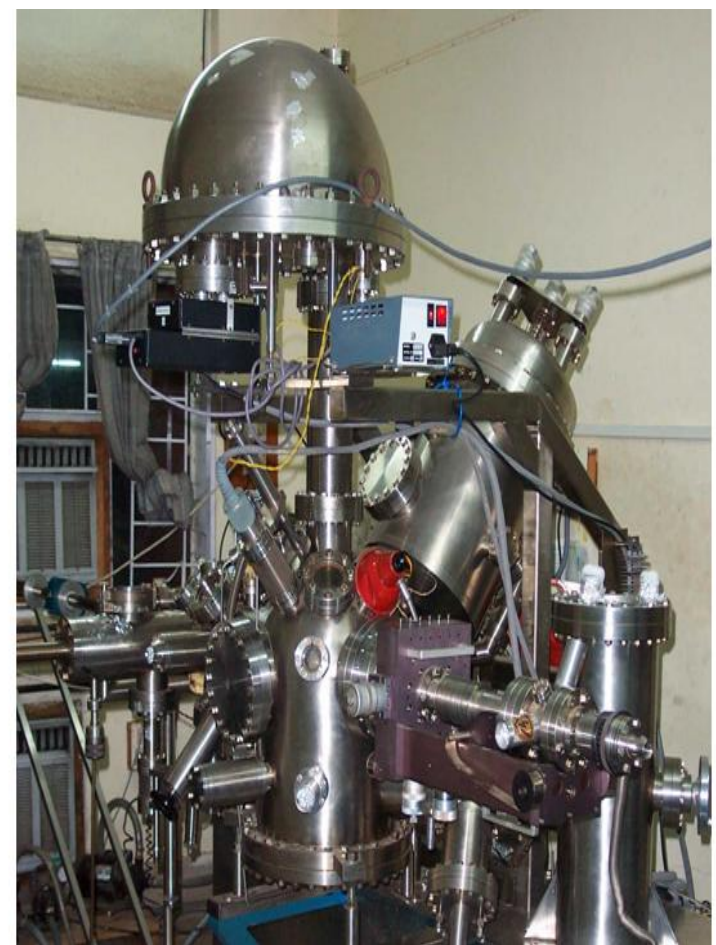

Fig. 4 Photograph of XPS equipment

Since for each element there is a characteristic binding energy of an electron associated with specific atomic orbital (s, p, d, f, etc.), by using the XPS measurement one can identify the elements present in the material. The concentration of them can also be measured since the intensity of the photoelectron lines is taken as the measure of this quantity. Due to the Coulombic interaction between electron and its surrounding there is an important effect of atomic environment on binding energy of an electron. This interaction results in shifting in binding energy of electrons and give rises to the identification of bonds on an atom constructing with surrounding atoms and subsequently revealing the compounds present in the structure [5].

For the non-conducting materials, a positive charge is built up in the sample surface following to the ejection of electrons from the specimen under X-ray irradiation and results in lowering the kinetic energy of liberated electrons due to the Coulombic interaction taking place between surface charge and electrons. To eliminate the effect of surface charging on shifting binding or kinetic energy, the correction is done based on the calibration of the binding energies with known reference binding energy of photoelectron line (in general, C 1s $(284.6 \mathrm{eV})$ ).

The identification of chemical states of determined element is accomplished by comparing the calculated binding energies of each component with reported state values by taking the chemical shift into account. Furthermore, the relative amount of present phases in specimen is revealed from the relative area under each component state. So, following all these complex analyzing stages, XPS measurement can reveal important surface properties of a material like the identification of elements present at the surface, chemical state of elements, quantity of chemical state of each element, spatial distribution of the material in 3-D, and concentration of element present in the material [5].

\section{ENERGY DISPERSIVE X-RAY ANALYSIS (EDXA) / SCANNING ELECTRON MICROSCOPY(SEM)}

Scanning electron microscopy (SEM) is a strong tool for the determination of structural, physical and compositional properties of solid state materials and devices [7-8]. A typical SEM system consists of an electron gun (creating electron beam), sample chamber (providing electron beam-sample interaction), several types of detectors for different applications (for detecting signals resulting from the beam-specimen interaction), and monitoring system (performing the image), which is schematically presented in Fig.5

When the specimen is bombarded by accelerated high energetic electrons, it results in a series of interaction with the nuclei and electrons of the specimen. This interaction give rise to change in direction of incident electrons (scattering) and gradual lose of energy of electrons. Scattering may be both elastic and inelastic, which results in deflection of incoming electrons by nuclei of atoms called backscattered electrons and generation of different signals such as emitting secondary electrons, electron-hole pairs, Auger electrons and characteristic Xrays, respectively [7-10]. The penetration depth of incident electrons depends on accelerating voltage and increases with increasing energy of incident electrons. 
In addition, it is known that the volume of interaction varies inversely with the atomic number of the sample determined [7-9]. Electron-specimen interaction origin generated secondary electrons, back scattered electrons, and characteristic X-rays provide surface morphology, composition contrast, and elemental analysis, respectively [9].
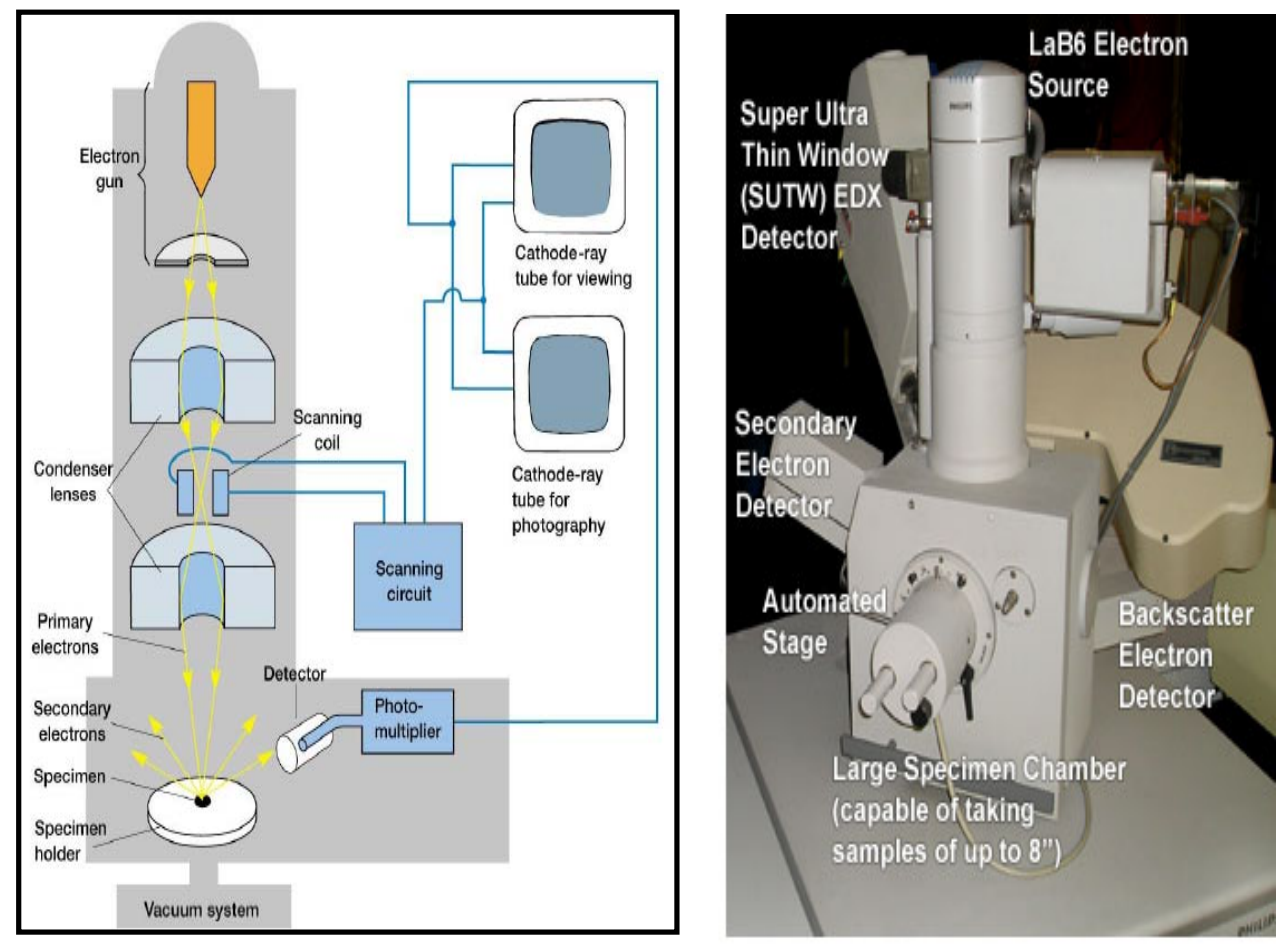

Fig.5 Diagram of Scanning Electron Microscope

A SEM equipped with energy dispersive X-ray analysis (EDXA) component determines the chemical composition of material on the micro- and nano scale by measuring the X-rays emitted during the electronspecimen interaction. The origin of the emitted characteristic X-rays is the change in the speed of bombarded electrons. By measuring the energies of X-rays emitted from the excited sample, the elements present in this region are qualitatively determined. The relative intensity of these characteristic X-rays is the measure of the atomic percentage of present constituent elements in the materials. So, by scanning the over all sample area it is possible to reveal spatial elemental mapping of the material.

\section{TRANSMISSION ELECTRON MICROSCOPY}

Transmission Electron Microscopy (TEM) has been used for the evaluation of the additive dispersion in the studied semiconductor oxides. Basic results about semiconductor nanoparticles size have been obtained using conventional TEM under bright-field conditions [9]. Under these conditions, we could also observe the macroagglomerated metals, with cluster sizes as big as the semiconductor nanoparticles size. In order to study material structure and morphology we have used High Resolution Electron Microscopy (HRTEM). This technique allows us to obtain images of the atomic structure of the materials studied. At the present time, modern electron microscopy can give resolutions of less than $2 \AA$, which is sufficient in order to characterize cluster cores of the heavier transition metals at atomic resolution [26]. However, HRTEM have a disadvantage: since the energy of the electron beam can be as much as $300 \mathrm{keV}$, the particles are heated up rapidly and rearrangement and coalescence processes may result.

\subsection{SAMPLE AND GRID PREPARATION}

In comparison with other materials, powder samples are the easiest to prepare. The greatest advantage is that grain size is normally thin enough to let the electrons pass through material, and no thinning is needed. There are two important parameters which should be improved in order to obtain good images of the nanoparticles. It should be assured a good dispersion of the nanopowders, avoiding their agglomeration. The second is to obtain grid membranes as thin as possible to improve TEM image. The sample preparing steps are explained schematically in fig.6 


\subsection{BRIGHT FIELD TEM}

As explained by other authors [9], bright field images can provide images with a good contrast. This property was useful to measure the particle and cluster sizes. In bright field conditions, the direct electron beam (not scattered) from the diffraction pattern has been selected with the objective aperture. If the image is formed with a wide objective aperture the contrast of the image will be poor because many beams contribute to the image. All the possible aspects concerning this technique are extensively reported in literature [7-11].

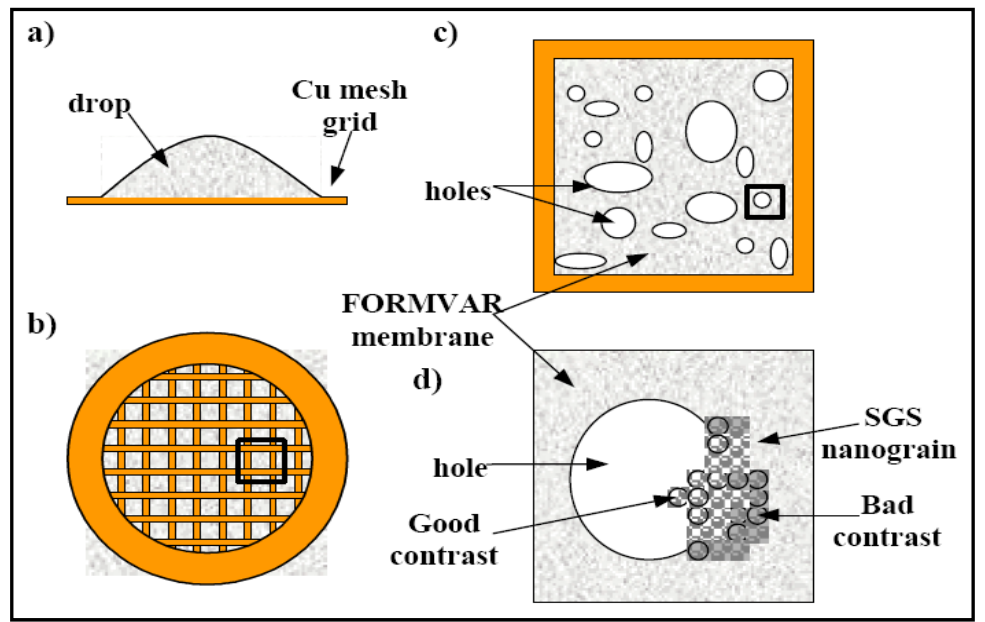

Fig.6 a) A drop containing the mixture of ethanol and our $\mathrm{Pd} / \mathrm{SnO}_{2}$ is deposited on a $\mathrm{Cu}$ mesh grid. b) After ethanol evaporation, the material remains in the FORMVAR membrane with holes. c) Magnified detail of the membrane d) $\mathrm{Pd} / \mathrm{SnO}_{2}$ nanopowders suspended on a hole will display good contrast HRTEM images.

\section{ATOMIC FORCE MICROSCOPE (AFM)}

The atomic force microscope (AFM), or scanning force microscope (SFM) was invented in 1986 by Binnig, Quate and Gerbe [12-14].AFM has been used in many applications like

\section{* Insulator surface topography}

* Charge deposition on insulator

* Atom by atom deposition

* Nanometer scale imaging for bio-samples where electron microscope is not suitable for preparatory reasons

* Measurement of roughness and hardness of specimen etc.

AFM consist of different parts as
1. Cantilever and Tip
2. Deflection sensing system
3. Photodiode
4. Piezoelectric scanner
5. High gain voltage amplifier
6. Vibration isolator

Each of these is integral part of AFM and has some specific role in carrying out measurement. AFM can be operated in three modes. They are

1. Contact mode

2. Non-contact mode

3. Tapping mode.

Like all other scanning probe microscopes, the AFM utilizes a sharp probe moving over the surface of a sample in a raster scan. In the case of the AFM, the probe is a tip on the end of a cantilever, which bends in response to the force between the tip and the sample as shown in fig.7. It measures the force interaction between a sharp tip and the surface and, since the force interaction does not depend on electrically conducting samples and tips, AFM can be applied to insulators as well. 


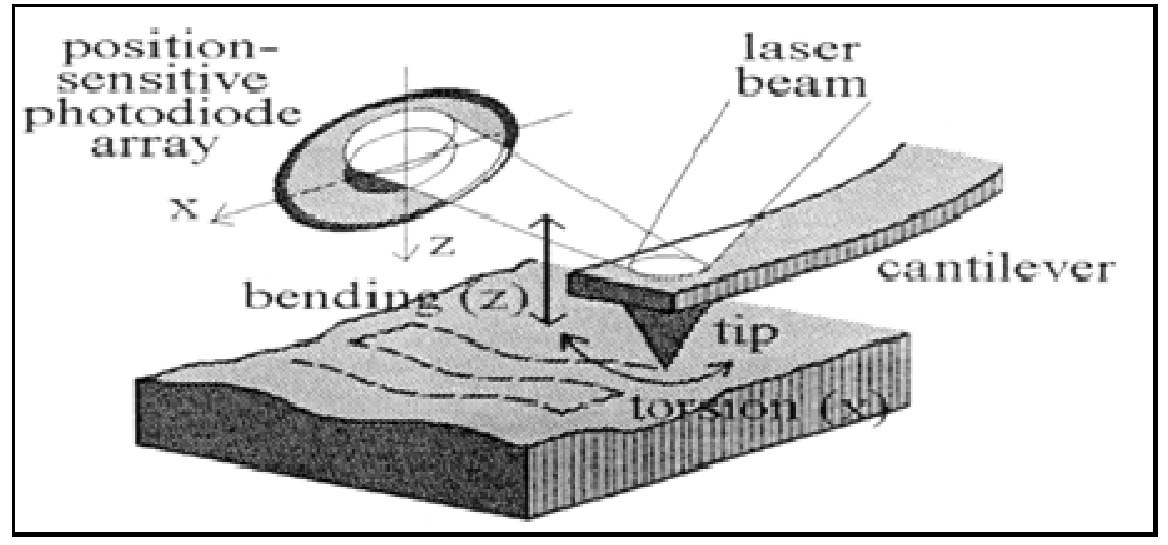

Fig.7 Diagram showing the most common way of measuring AFM cantilever deflection

In order to measure these forces, a probe tip is mounted on a cantilever-type spring. Then, the force interaction between the sample and the tip after approaching each other causes the cantilever to deflect according to

$$
\text { Hooke’ s law } \mathrm{F}=\mathrm{c} \cdot \Delta \mathrm{z}
$$

where $\mathrm{c}$ is the spring constant of the tip and $\Delta \mathrm{z}$ is the deflection. These deflections can be sensed by various means. One of the most common is laser beam deflection as shown in fig 7

\section{SHEET RESISTIVITY MEASUREMENT}

Sheet resistance, an electrical property of thin films was measured at room temperature using four probe and Keitheley meters. A schematic of a four point probe is shown in fig. 6 In this technique four probes are gently placed on the thin film sample. A constant current is made to flow through the length of the sample through probes labeled 1 and 4 in the fig. 8 If the sample has any resistance to the flow of electrical current, then there will be a drop of potential as the current flows along the sample, between the probes 2 and 3 . The voltage drop between probes 2 and 3 can be measured by a digital voltmeter. The high impedance of the digital voltmeter minimizes the current flow through the portion of the circuit comprising the voltmeter. Since there is no potential drop across the contact resistance associated with probes 2 and 3, only the resistance associated with the thin film between probes 2 and 3 is been measured [15].

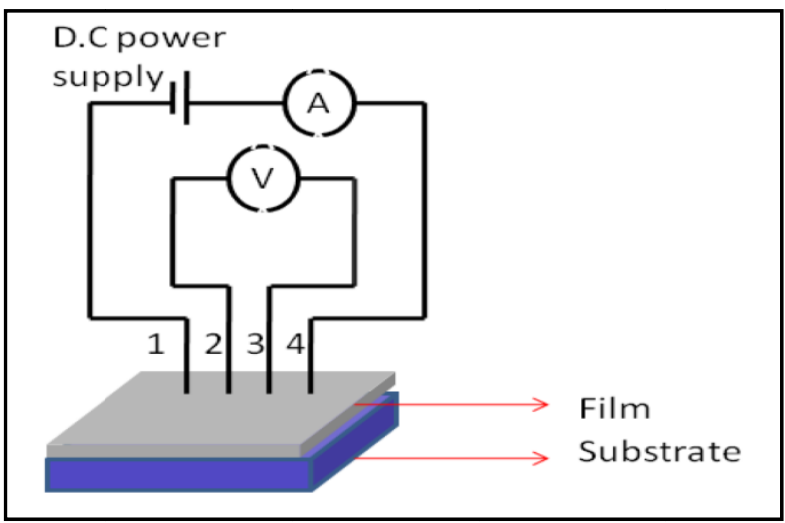

Fig.8 schematic diagram of a inline four probe measuring setup

The sheet resistance of the $\mathrm{SnO}_{2}$ films deposited on silicon substrates was measured by four probe technique using NAGY sheet resistance meter (SD-510). The measuring probe (SDKR-25) consists of four rhodium plated in line spring loaded tips, each tip separated by $1 \mathrm{~mm}$ from each other. The sheet resistance of the film was measured at room temperature by carefully mounting the probe on the sample. And the digital meter in the instrument reads the sheet resistance value of the films directly. 


\section{VII.THICKNESS MEASUREMENTS OF THIN FILMS}
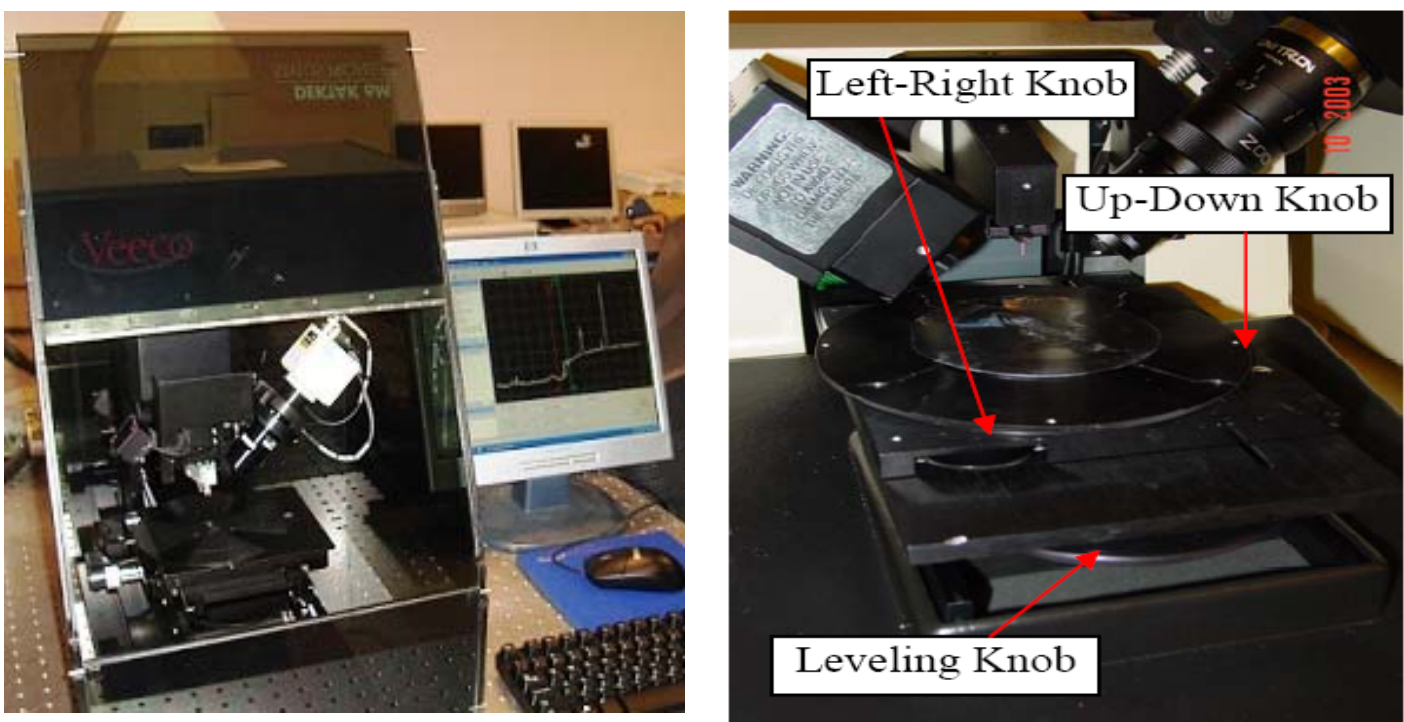

Fig.9 Dektak Stylus equipment used for film thickness measurement

The equipment named Dektak is used to measure the thickness or roughness of thin films, in the nanometer range with high repeatability. The Dektak has a large stage where a sample is placed underneath a stylus. The stage has an X-Y movement of approximately 2 inches. A 640 x 480-pixel (1/3 inch format) USB video camera is fitted with a fixed magnification, with an adjustable light source to help when viewing samples with different reflectivity. The stylus has a diamond tip which is moved over the surface of a material electromechanically taking measurements of the sample moving underneath it. As the sample is moved, the stylus is moved over the surface, making vertical movements. As it does this, an electrical signal corresponding to the vertical movement of the stylus is produced, allowing for measurement of film thickness/roughness. This method allows for highly accurate and reproducible measurements in two dimensions. The Dektak can also be programmed to make a three dimensional map of a surface to allow for viewing of film roughness over an area of the film.

\section{FOURIER TRANSFORM INFRARED SPECTROSCOPY (FTIR)}

FTIR is an effective analytical tool for identification of unknown sample. The principle of FTIR is that the gas/solid/liquid to be analyzed is led through a cuvette with an IR light source at one end that is sending out scattered IR light, and a modulator that "cuts" the infra-red light into different wave lengths. At the other end of the cuvette a detector is measuring the amount of IR light to pass through the cuvette.

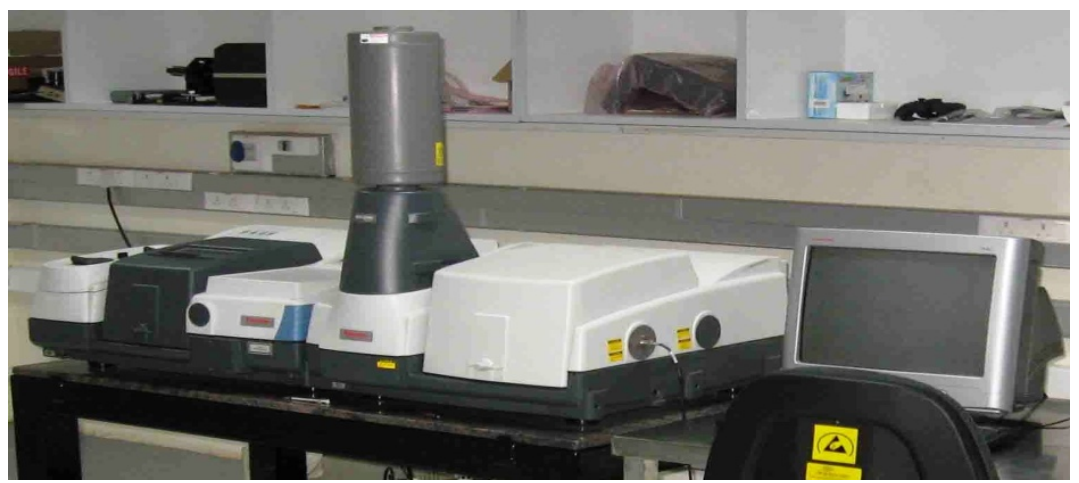

Fig.10 Photograph of FTIR Instrument

Since chemical bonds absorb infrared energy at specific frequencies (or wavelengths), the basic structure of compounds can be determined by the spectral locations of their IR absorptions. The plot of a compound's IR transmission vs. frequency is its "fingerprint", which when compared to reference spectra identifies the material. FTIR relies on the fact that the most molecules absorb light in the infra-red region of the electromagnetic spectrum; this absorption corresponds specifically to the bonds present in the molecule. The sample is irradiated by a broad spectrum of infra-red light and the level of absorbance at a particular frequency is plotted after Fourier transforming the data. The resulting spectrum is characteristic of the organic molecules present in the sample[17-18]. 


\subsection{SPECTROSCOPIC ELLIPSOMETRY}

The basic principle of ellipsometer depend upon the fact that linearly polarized incident light has reflection coefficients which depend on the direction of polarization. The two polarization directions of interest are the $p$ or TM (electric field parallel to the plane of incidence) and the s or TE (electric field perpendicular to the plane of incidence). Linearly polarized light is decomposed into the $\mathrm{p}$ and s components as shown in fig. 10

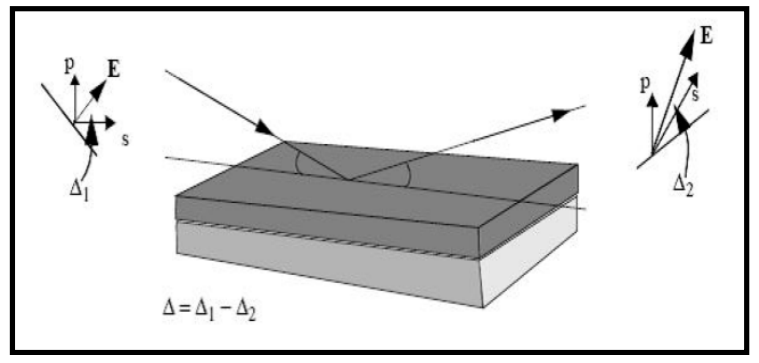

Fig.11: Spectroscopic Ellipsometry measurements

These component waves experience different amplitude attenuations, and different absolute phase shifts upon reflection; hence, the state of polarization is changed. Ellipsometry refers to the measurement of the state of polarization before and after reflection for the purpose of studying the properties of the reflecting boundary and also finding the thickness of the films. [19-20].
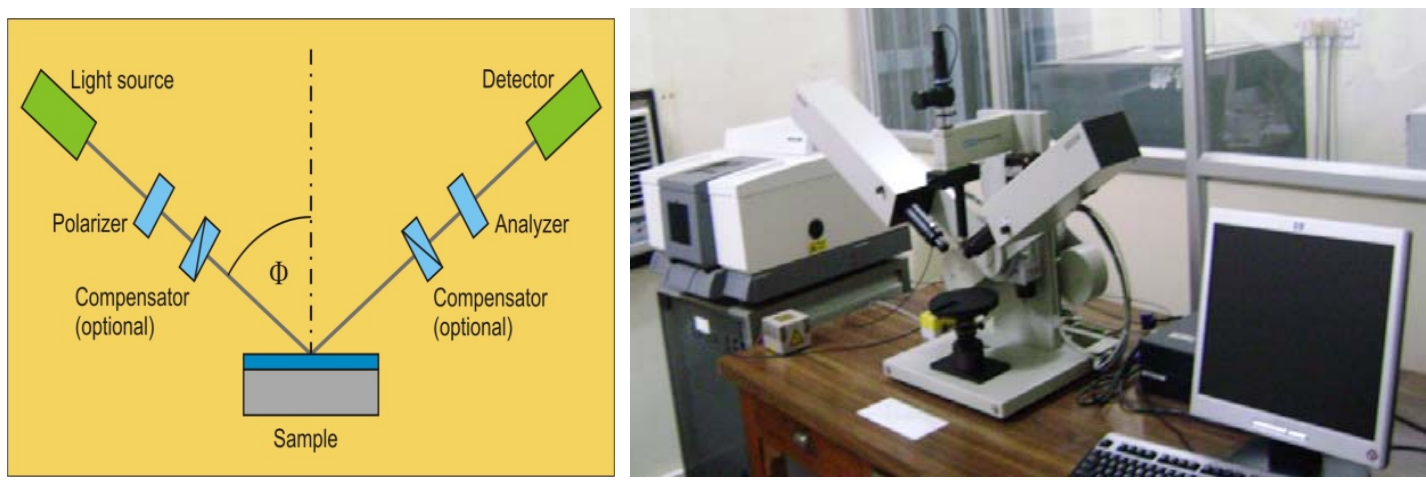

Fig.12 Schematic diagram and picture of Ellipsometry equipment

\section{ELECTRICAL SENSITIVITY MEASUREMENT}

For gas sensing test, the sensor device called as chemiresistor and measurement system were designed using the concept of resistance variation in the sensing materials after the target gas exposure. The gas sensing behavior of chemiresistor sensors was studied by calculating the change in the surface resistance of sensing film with time towards the exposure of the target gas (step by step varying the concentration) at a particular pressure with a fixed temperature. The resistance variation was measured by Keitheley $2410 \mathrm{C}$ model source meter which has $0.012 \%$ basic accuracy with $5 \frac{1}{2} 2$-digit resolution.

The chemiresistor type sensors were mounted on the hot plate (If it is an integrated gas sensor, microheater will be incorporated with the gas sensing film itself.) which was coupled with ceramic base stand. The two leads of thin silver wire were attached to interdigitated electrodes by silver paste for electrical connections. Finally this sensor setup was fixed into the homemade iron gas sensing chamber. The electrical connections for gas sensing measurements, thermocouple and temperature variation were made using instrumentation feed through. The schematic block diagram of gas sensor setup is shown in Fig. 13 and the in house fabricated gas sensing setup is shown in fig $14 \&$ Fig. 15

During the sensing measurement, initially sensing chamber was cleaned by connecting it with a low vacuum rotary pump. The sensor was exposed to pure air until the constant baseline resistance was achieved and then the gas analytes was introduced in the chamber and the resistance of the sensor was recorded for every $10 \mathrm{mins}$ or so. During this time the steady resistance had been achieved, and then the sensing chamber was flushed with pure air consecutively to allow the surface of the sensitive film to regain atmospheric condition and the resistance of it reached steady and kept stable. The response of sensor was monitored in terms of the normalized resistance calculated by Response $\left(\mathrm{R}_{0} / \mathrm{R}_{\mathrm{g}}\right)$ and the sensitivity factor was monitored. $\mathrm{R}_{\mathrm{g}}$ is the resistance of the sensor in the presence of analytes gas and $R_{0}$ is the initial baseline resistance of the films. 


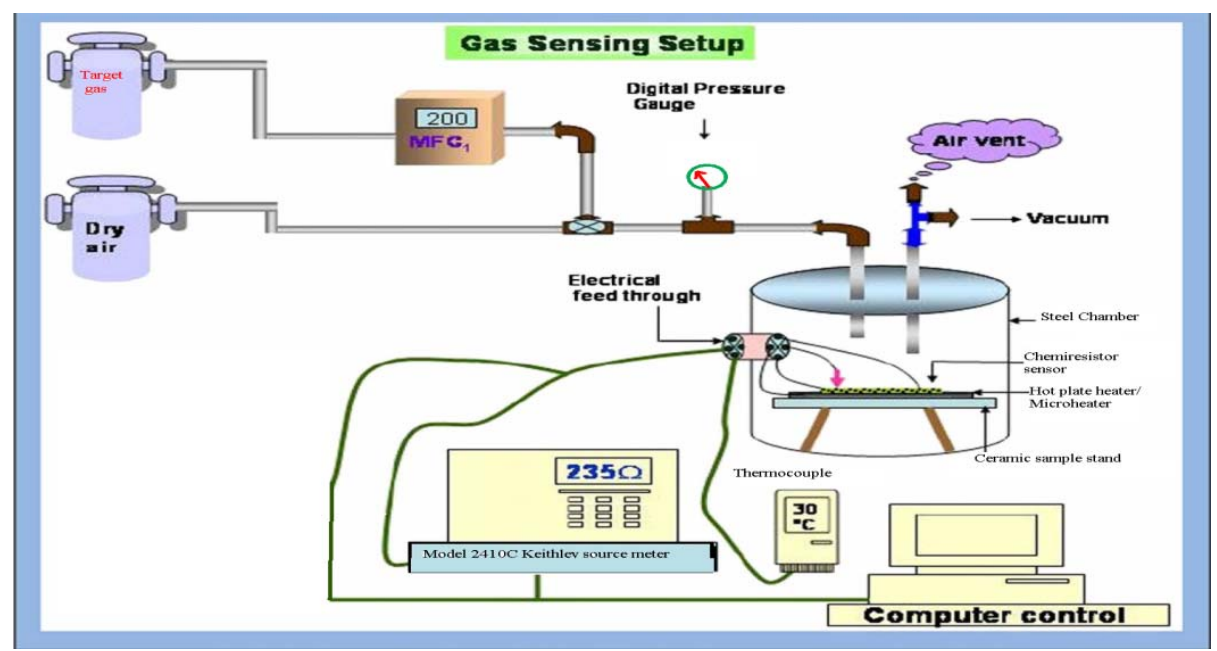

Fig. 13 Schematic block diagram of gas sensing setup used

Once the resistance changes are noted down in the gas sensing experiments, to get the sensitivity analysis the sensitivity response curves had been drawn based on the schematic shown in fig 14 and fig. 15

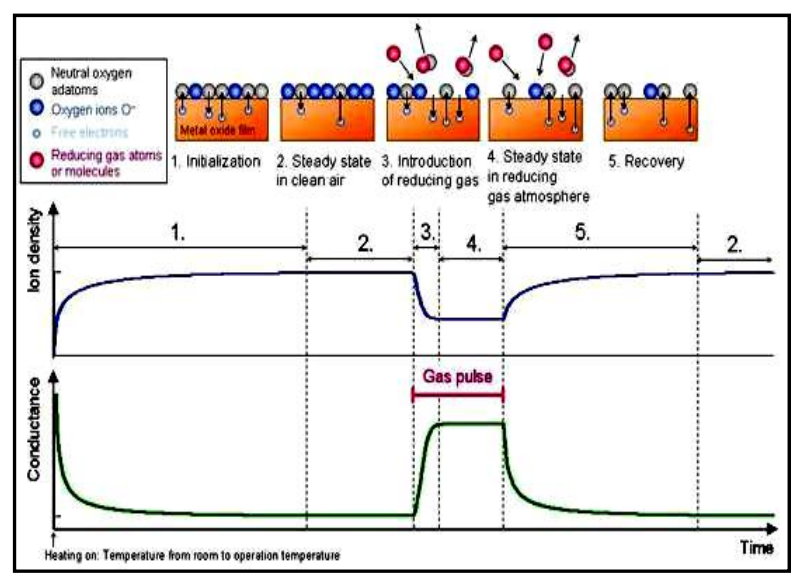

Fig.14: A schematic response curve of gas sensor testing. The typical characteristic values extracted from the measurement are identified on the plots.

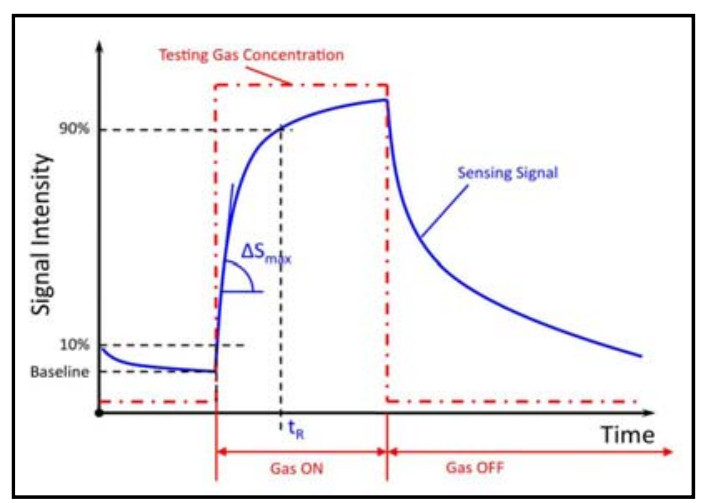

Fig.15 A schematic of a typical response curve of gas sensor testing 


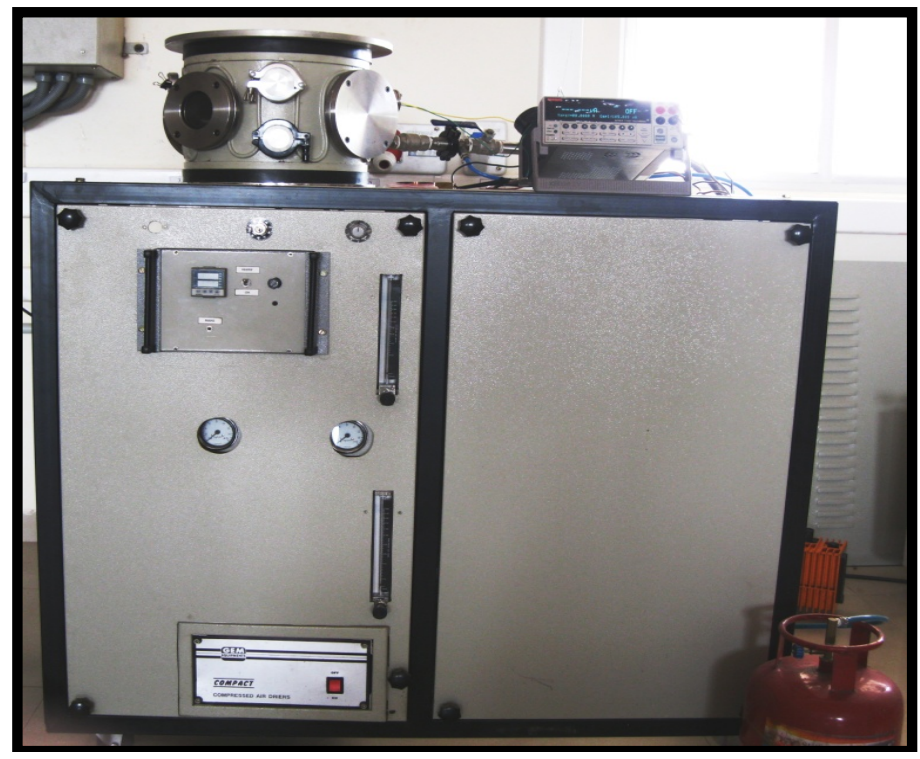

Fig 16 Gas sensor setup used for LPG sensitivity measurement

\section{REFERENCES}

[1] C. S. Barret, "Structure of Metals, Crystallographic Methods, Principles and Data". Mc Graw-Hill, New York, 1956.

[2] B. D. Cullity, "Elements of X-ray Diffraction". Addison-Wesley, Second Edition, 1978.

[3] C. Suryanarayana, M. Grant Norton, "X-ray Diffraction: a practical approach". Plenum Press, New York, 1998.

[4] C. R. Brundle, C. A. Evans, shaun Wilson, "Encylopedia of Materials Characterization: Surfaces, Interfaces", Thin Films. Butterworth- Heinemman, Elsevier, 1992.

[5] ohn F. Watts, J. Wolstenholme, "An Introduction to Surface Analysis by XPS and AES”. John Willey and Sons Ltd, 2003.

[6] B. G. Yacobi, "Semiconductor Materials: An Introduction to Basic Principles". Kluwer Academic/Plenum Publishers, London 2002.

[7] Goldstein, J. (2003) "Scanning electron microscopy and x-ray microanalysis". Kluwer Adacemic/Plenum Pulbishers, 689 p.

[8] Reimer, L. (1998) "Scanning electron microscopy : physics of image formation and microanalysis". Springer, 527 p.

[9] Egerton, R. F. (2005) "Physical principles of electron microscopy : an introduction to TEM, SEM, and AEM". Springer, 202.

[10] Clarke, A. R. (2002) "Microscopy techniques for materials science". CRC Press

[11] S. L. Flegler, J. W. Heckmen, K. L. Klomparens, "Scanning and Transmission Electron Microscopy, An introduction", Oxford University Press. Oxford, 1993.

[12] Giessibl, Franz J. (2003). "Advances in atomic force microscopy".Reviews of Modern Physics 75: 949

[13] Geisse, Nicholas A. (July-August 2009). "AFM and Combined Optical Techniques". Materials Today 12 (7-8): 40-45.

[14] Butt, H; Cappella, B; Kappl, M (2005). "Force measurements with the atomic force microscope: Technique, interpretation and applications". Surface Science Reports 59: 1-152

[15] D. K. Schroder, "Semiconductor Material and Device Characterization", A wiley- Interscience Publication, John Wiley \& Sons, Inc (1990).

[16] www.veeco.com

[17] F. Abeles "Optical-Properties of Very Thin-Films" Thin Solid Films, 34 (1976), pp. 291-302

[18] T.W.H. Oates a, H. Wormeester, H. Arwin, "Characterization of plasmonic effects in thin films and metamaterials using spectroscopic Ellipsometry, Progress in Surface Science 86 (2011) 328-376

[19] Yeon Sik Jung, "Spectroscopic ellipsometry studies on the optical constants of indium tin oxide films deposited under various sputtering conditions", Thin Solid Films 467, 36-42 (2004).

[20] E.A.Irene, "Applications of Spectroscopic Ellipsometry to Microelectronics", Thin Solid Films, 233, 96-111 (1993). 